\title{
Sonography of Cat Scratch Disease
}

\author{
David M. Melville, MD, Jon A. Jacobson, MD, Brian Downie, PA-C, MS, J. Sybil Biermann, MD, \\ Sung Moon Kim, MD, Corrie M. Yablon, MD
}

Received April 8, 2014, from the Departments of Radiology (D.M.M., J.A.J., B.D., S.M.K., C.M.Y.) and Orthopedic Surgery (J.S.B.), University of Michigan, Ann Arbor, Michigan USA; and Department of Medical Imaging, University of Arizona Health Sciences Center, Tucson, Arizona USA (D.M.M.). Revision requested June 4, 2014. Revised manuscript accepted for publication June 16, 2014.

We thank Elizabeth Krupinksi, $\mathrm{PhD}$, for assistance.

Address correspondence to David M. Melville, MD, Department of Medical Imaging, University of Arizona Health Network, $1501 \mathrm{~N}$ Campbell Ave, PO Box 245067, Tucson, AZ 85724 USA.

E-mail:dmelville@radiology.arizona.edu

Abbreviations

MRI, magnetic resonance imaging

doi:10.7863/ultra.34.3.387
Objectives - To characterize the sonographic features of cat scratch disease and to identify features that allow differentiation from other causes of medial epitrochlear masses.

Methods - After Institutional Review Board approval was obtained, patients who underwent sonography for a medial epitrochlear mass or lymph node were identified via the radiology information system. Patients were divided into 2 groups: cat scratch disease and non-cat scratch disease, based on pathologic results and clinical information. Sonograms were retrospectively reviewed and characterized with respect to dimension, shape (round, oval, or lobular), symmetry, location (subcutaneous or intramuscular), multiplicity, echogenicity (anechoic, hypoechoic, isoechoic, hyperechoic, or mixed), hyperechoic hilum (present or absent), adjacent anechoic or hypoechoic area, hyperemia (present or absent), pattern of hyperemia if present (central, peripheral, or mixed), increased posterior through-transmission (present or absent), and shadowing (present or absent). Sonographic findings were compared between the patients with and without cat scratch disease.

Results - The final patient group consisted of 5 cases of cat scratch disease and 16 cases of other causes of medial epitrochlear masses. The 2 sonographic findings that were significantly different between the cat scratch disease and non-cat scratch disease cases included mass asymmetry $(P=.0062)$ and the presence of a hyperechoic hilum $(P=.0075)$. The other sonographic findings showed no significant differences between the groups.

Conclusions - The sonographic finding of an epitrochlear mass due to cat scratch disease most commonly is that of a hypoechoic lobular or oval mass with central hyperemia and a possible adjacent fluid collection; however, the presence of asymmetry and a hyperechoic hilum differentiate cat scratch disease from other etiologies.

Key Words — cat scratch disease; epitrochlear; lymph node; mass; musculoskeletal ultrasound; sonography

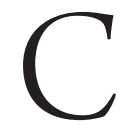
at scratch disease is typically a self-limiting infectious condition, often presenting in children and adolescents as a benign regional lymphadenitis that results from a cat scratch or bite involving the distal upper extremity. ${ }^{1}$ The initial trauma results in inoculation with Bartonella henselae and subsequent regional lymph node enlargement, most commonly the medial epitrochlear region of the elbow. ${ }^{2-5}$ The development of a palpable mass may raise clinical concern for a soft tissue neoplasm, such as sarcoma, lymphoma, or metastatic disease; therefore, imaging evaluation is often considered. ${ }^{6-9}$ 
Medial epitrochlear lymphadenopathy from cat scratch disease has been characterized on magnetic resonance imaging (MRI) as enlarged enhancing lymph nodes with possible necrosis and surrounding edema. ${ }^{10,11}$ Magnetic resonance imaging findings may appear nonspecific and simulate malignancy. The sonographic appearance of epitrochlear lymphadenopathy from cat scratch disease has been described as hypoechoic enlarged lymph nodes with possible through-transmission and substantial hyperemia. ${ }^{12,13}$ Accurate characterization of lymph node enlargement in cat scratch disease would be important to help differentiate lymphadenopathy of cat scratch disease from other etiologies, which may direct appropriate confirmatory serologic testing and prevent unnecessary surgical procedures and additional imaging.

In our clinical practice, in contrast to what has been described in the literature, we have noted cases of cat scratch disease in which enlarged lymph nodes retained a hyperechoic hilum with an adjacent fluid collection. The purpose of this study was to retrospectively characterize the sonographic appearances of abnormal epitrochlear masses from cat scratch disease and to compare such findings to other causes of medial epitrochlear masses and lymphadenopathy.

\section{Materials and Methods}

Institutional Review Board approval was obtained and informed consent waived. Sonographic reports in the radiology information system from January 1, 2000, through May 1, 2013, were searched to identify patients with epitrochlear masses by using key terms, including "cat scratch disease," "epitrochlear mass," "epitrochlear node," "elbow," "lymph node," and "elbow biopsy." Patient medical records were reviewed, including other correlative imaging findings, pathologic results, laboratory analysis, as well clinical and surgical histories.

Sonograms of the elbow were obtained during both diagnostic examinations and sonographically guided biopsies as part of routine clinical practice at our institution using high-frequency transducers $(10-17 \mathrm{MHz})$ on 1 of 4 clinical machines (iU22 and HDI 5000; Philips Healthcare, Bothell, WA; and LOGIQE9 and LOGIQ9; GE Healthcare, Milwaukee, WI). Images were acquired in transverse and longitudinal orientations with routine use of color and power Doppler imaging. Static images and cine clips were stored on the department's picture archiving and communication system.

A retrospective consensus review of sonograms from the final study group was then performed by 2 fellowship- trained musculoskeletal radiologists (with 1 and 16 years of experience, respectively) who were blinded to the final diagnosis. Static and cine images were reviewed, and the dominant epitrochlear mass was evaluated for largest dimension (centimeters), overall shape (round, oval, or lobular), symmetry with respect to shape (uniform or asymmetrically enlarged component), location (subcutaneous or intramuscular), multiplicity, echogenicity with respect to adjacent subcutaneous tissue (anechoic, hypoechoic, isoechoic, hyperechoic, or mixed), hyperechoic hilum (present or absent), presence of an adjacent anechoic or hypoechoic area, hyperemia (present or absent), pattern of hyperemia if present (central, peripheral, or mixed), increased posterior through-transmission (present or absent), and shadowing (present or absent).

These data were then evaluated to determine whether any of the sonographic characteristics permitted differentiation of epitrochlear soft tissue masses due to cat scratch disease from other benign and malignant etiologies using nonparametric analysis in the form of the Mann-Whitney $U$ test (SAS 9.2 software; SAS Institute Inc, Cary, NC). $\mathrm{A} \chi^{2}$ test was used for all categorical variables. In addition, sonograms were correlated with MRI and computed tomography when available.

\section{Results}

The initial key word search identified 86 patients undergoing sonography for an elbow mass, of which 21 had a diagnosis for the mass on the basis of pathologic results, laboratory parameters, and clinical presentation. The 65 excluded cases either had no mass at imaging or were without a diagnosis. The final study group of 21 patients consisted of $57 \%$ female patients (12 of 21 ) and $43 \%$ male patients ( 9 of 21) with an average age of 37 years (range, 5 94 years). The right elbow was involved in $62 \%$ ( 16 of 26) and the left in $38 \%$ ( 10 of 26 ).

Of these 21 patients, $24 \%$ ( 5 of 21 ) had a diagnosis of and were treated for cat scratch disease, and 76\% (16 of 21) had epitrochlear elbow masses not representing cat scratch disease, which consisted of both benign and malignant etiologies, including angiolipoma (1), a desmoid (1), granuloma annulare (1), a reactive lymph node (2), a rheumatoid nodule (1), a ruptured epidermoid cyst (1), sarcoidosis (2), sarcoma (3), schwannoma (3), and a solitary fibrous tumor (1). All non-cat scratch disease diagnoses were established by percutaneous biopsy or surgical removal and pathologic evaluation.

The 5 cases identified as cat scratch disease consisted of $60 \%$ female patients ( 3 of 5 ) and $40 \%$ male patients ( 2 
of 6) with an average age of 20 years (range, 11-28 years), which was outside the range of peak incidence. The right elbow was involved in $60 \%$ (3 of 5) and the left in $40 \%$ (2 of 5), with ipsilateral axillary lymph node involvement in $20 \%$ ( 1 of 5 ). Two of the 5 cases of cat scratch disease were diagnosed by positive $B$ henselae titers, and 2 were diagnosed by percutaneous biopsy, which showed reactive tissue with necrosis and granuloma formation combined with a history of cat exposure. The final case was presumptively diagnosed on the basis of clinical symptoms, multiple cat exposures, and spontaneous regression of lymphadenopathy. No cases underwent surgical excision. Contact with cats was reported in $80 \%$ ( 4 of 5 ), with 2 patients reporting owning a single cat, 1 patient reporting 2 cats, and 1 patient reporting "several cats," although the exact total number of cats in this single household could not be documented. The history of a scratch and the presence of a wound were noted in $40 \%$ of cases ( 2 of 5 ).

Retrospective sonographic review of the 5 cases of cat scratch disease (Table 1) showed that the average size of the dominant medial epitrochlear mass was $2.5 \mathrm{~cm}$ (range, $1.9-3.6 \mathrm{~cm}$ ). Its shape was oval in $60 \%$ ( 3 of 5 ) and lobular in $40 \%$ ( 2 of 5), and it was asymmetric in all cases (Figures $1-3)$. The location of the dominant mass was subcutaneous in $100 \%$ ( 5 of 5). There were multiple masses in $80 \%$ ( 4 of $5)$. The dominant mass was predominantly hypoechoic in $80 \%$ ( 4 of 5) and isoechoic in 20\% ( 1 of 5), with a hyperechoic hilum shown in 60\% (3 of 5). An adjacent anechoic or hypoechoic area was seen in 40\% (2 of 5) and, when present, correlated with MRI findings (Figures 2 and 3 ). Hyperemia was seen in $60 \%$ ( 3 of 5 ), all of which showed a central blood flow pattern. Increased posterior through-transmission was seen in $20 \%$ ( 1 of 5), and none showed shadowing.
Retrospective sonographic review of the remaining 16 cases (not representing cat scratch disease; Table 1) showed that the average size of the dominant medial epitrochlear mass was $2.3 \mathrm{~cm}$ (range, $0.8-4.5 \mathrm{~cm}$ ). Its shape was oval in $63 \%$ (10 of 16$)$, lobular in $31 \%$ ( 5 of 16 ), and round in $6 \%$ (1 of 16), and it was symmetric in $75 \%$

Table 1. Sonographic Characteristics of Medial Epitrochlear Masses

\begin{tabular}{|c|c|c|c|}
\hline Parameter & $\begin{array}{l}\text { Cat Scratch } \\
\text { Disease }\end{array}$ & $\begin{array}{l}\text { Other } \\
\text { Masses }\end{array}$ & $P$ \\
\hline Patients & $24(5 / 21)$ & $76(16 / 21)$ & NA \\
\hline Average size (range), cm & $2.5(1.9-3.6)$ & $2.3(0.8-4.5)$ & .5687 \\
\hline \multicolumn{4}{|l|}{ Shape } \\
\hline Oval & $60(3 / 5)$ & $63(10 / 16)$ & $>.99$ \\
\hline Round & $0(0 / 5)$ & $6(1 / 16)$ & $>.99$ \\
\hline Lobular & $40(2 / 5)$ & $31(5 / 16)$ & $>.99$ \\
\hline Symmetric & $0(0 / 5)$ & $75(12 / 16)$ & .0062 \\
\hline \multicolumn{4}{|l|}{ Location } \\
\hline Subcutaneous & $100(5 / 5)$ & $94(15 / 16)$ & $>.99$ \\
\hline Intramuscular & $0(0 / 5)$ & $6(1 / 16)$ & $>.99$ \\
\hline Multiple & $80(4 / 5)$ & $12(2 / 16)$ & $>.99$ \\
\hline \multicolumn{4}{|l|}{ Echogenicity } \\
\hline Hypoechoic & $80(4 / 5)$ & $82(13 / 16)$ & $>.99$ \\
\hline Isoechoic & $20(1 / 5)$ & $6(1 / 16)$ & .4286 \\
\hline Hyperechoic & $0(0 / 5)$ & $12(2 / 16)$ & $>.99$ \\
\hline Hyperechoic hilum & $60(3 / 5)$ & $0(0 / 16)$ & .0075 \\
\hline \multicolumn{4}{|l|}{ Peripheral anechoic/ } \\
\hline Hyperemia & $60(3 / 5)$ & $50(8 / 16)$ & $>.99$ \\
\hline \multicolumn{4}{|l|}{ Flow pattern } \\
\hline Central & $100(3 / 3)$ & $25(2 / 8)$ & .063 \\
\hline Mixed & $0(0 / 0)$ & $75(6 / 8)$ & .2621 \\
\hline Through-transmission & $20(1 / 5)$ & $25(4 / 16)$ & $>.99$ \\
\hline Shadowing & $0(0 / 5)$ & $0(0 / 16)$ & NA \\
\hline
\end{tabular}

Data are presented as percent (number) where applicable. NA indicates not applicable.

Figure 1. Images from an 11-year-old girl with an epitrochlear mass from cat scratch disease. Grayscale (A) and color Doppler (B) long-axis sonograms show a hypoechoic lobular mass (arrows) that is asymmetric (arrowheads) with a hyperechoic central hilum (curved arrow) and central hyperemia. $U$ indicates ulnar nerve.

A

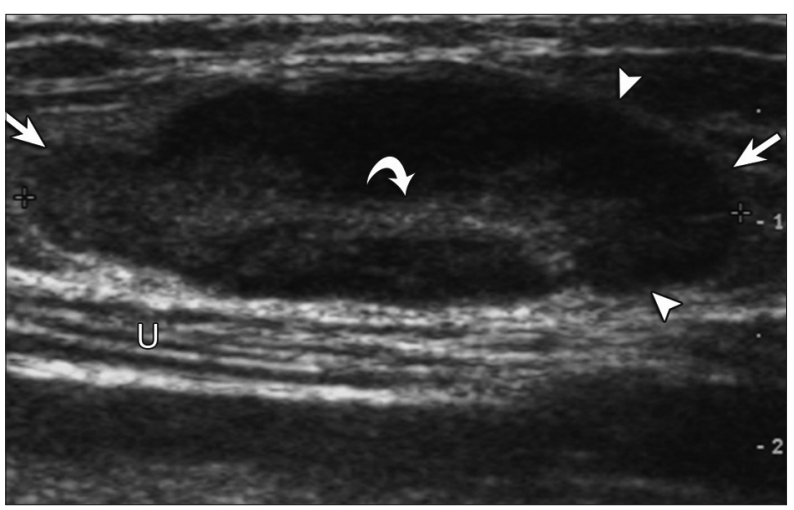

B

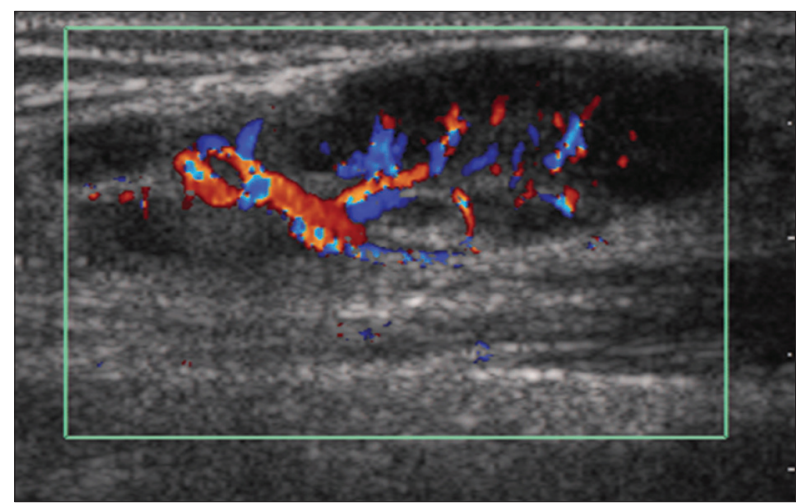


(12 of 16; Figures 4 and 5). The location of the dominant mass, which was multiple in $12 \%$ ( 2 of 16 ), was subcutaneous in $94 \%$ (15 of 16) and intramuscular in 6\% (1 of 16). The dominant mass was predominantly hypoechoic in $82 \%$ ( 13 of 16 ), hyperechoic in $12 \%$ ( 2 of 16 ), and isoechoic in 6\% (1 of 16). None of the masses showed a hyperechoic hilum. An adjacent anechoic or hypoechoic area was seen in 6\% ( 1 of 16). Hyperemia was seen in 50\% (8 of 16), which showed a central blood flow pattern in $25 \%$ (2 of 8) and a peripheral pattern in 75\% (6 of 8). Increased posterior through-transmission was seen in $25 \%$ (4 of 16), and none showed shadowing.

When comparing the sonographic findings between the cat scratch disease and non-cat scratch disease cases, there were significant differences $(P<.05)$ with regard to symmetry of the medial epitrochlear mass and the presence of a hyperechoic hilum; cases of cat scratch disease showed mass asymmetry and the presence of a hyperechoic hilum. Furthermore, the data were analyzed to determine whether there were significant differences in the various parameters measured as a function of a cat scratch disease versus noncat scratch disease diagnosis (Table 2). Once again, symmetry and a hyperechoic hilum were significant parameters $(P<.05)$; in addition, the presence of a peripheral anechoic or hypoechoic area was found to be statistically significant. Logistic regression for these 3 parameters with a cat scratch disease versus non-cat scratch disease diagnosis as the dependent variable indicated that asymmetry followed by a hyperechoic hilum and then a peripheral anechoic or hypoechoic region were the best predictors of a cat scratch disease versus non-cat scratch disease diagnosis $\left(r^{2}=0.783\right.$; $\log$ likelihood = 2.502).

Figure 2. Images from a 14-year-old girl with an epitrochlear mass from cat scratch disease. Grayscale sonogram (A) shows a hypoechoic lobular mass (curved arrow) and an adjacent complex fluid collection (arrowheads). T1-weighted (B), T2-weighted (C), and T1-weighted gadoliniumenhanced, fat-saturated (D) MR images show a corresponding soft tissue mass (curved arrows) and an adjacent ring-enhancing fluid collection (arrowheads)

A

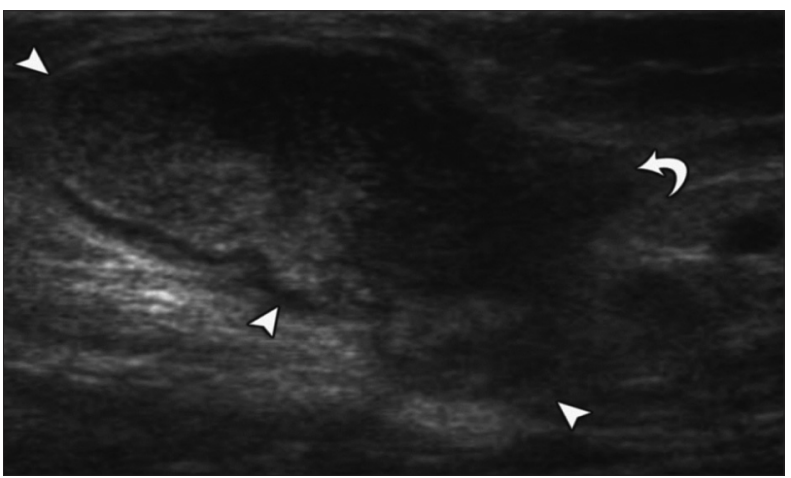

C

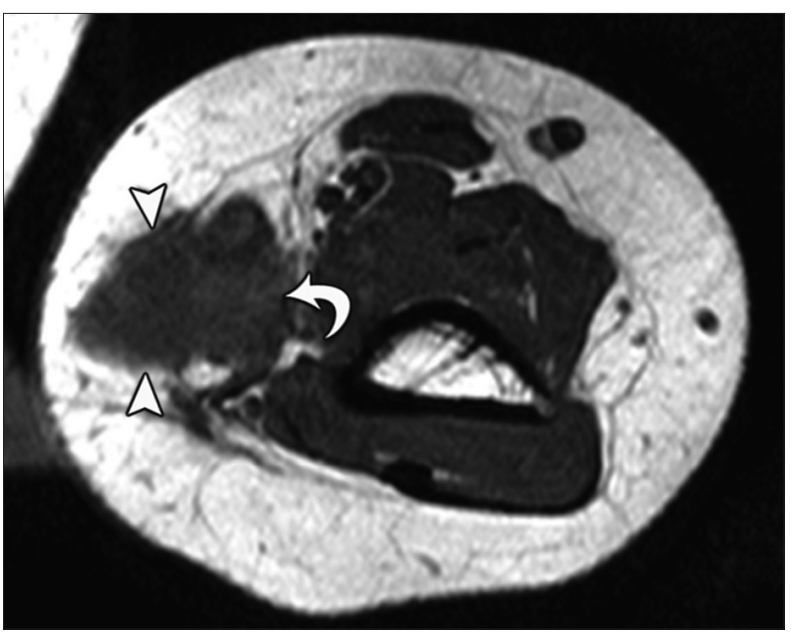

B

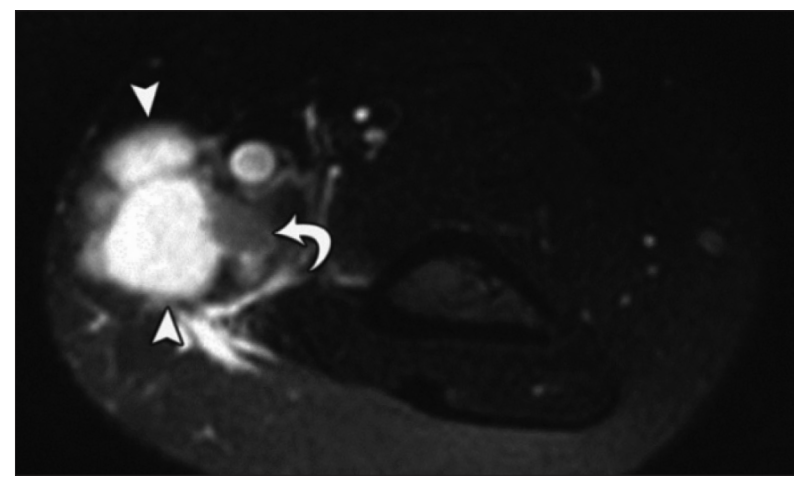

D

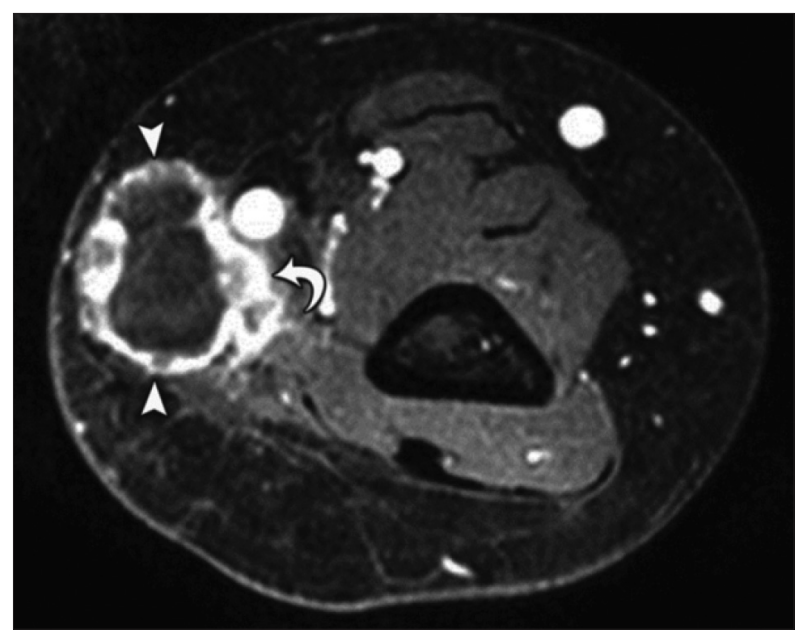




\section{Discussion}

Abnormal medial epitrochlear lymph node enlargement is a characteristic finding of cat scratch disease; however, imaging findings may appear confusing and potentially be misinterpreted for other disease types such as malignancy, leading to unnecessary biopsy. The results of our retrospective study showed that identification of a mass in the expected location of a medial epitrochlear lymph node that showed asymmetry and a hyperechoic hilum was charac-

Figure 3. Images from a 28-year-old man with an epitrochlear mass from cat scratch disease. Grayscale long-axis sonogram (A) shows an oval asymmetric hypoechoic mass (arrowheads) with a hyperechoic hilum (arrow) and an adjacent hypoechoic complex fluid collection (curved arrow). Proton density-weighted (B), T2-weighted (C), and T1-weighted gadolinium-enhanced, fat-saturated (D) MR images show a corresponding soft tissue mass (arrowheads) and an adjacent ring-enhancing fluid collection (curved arrows).

A

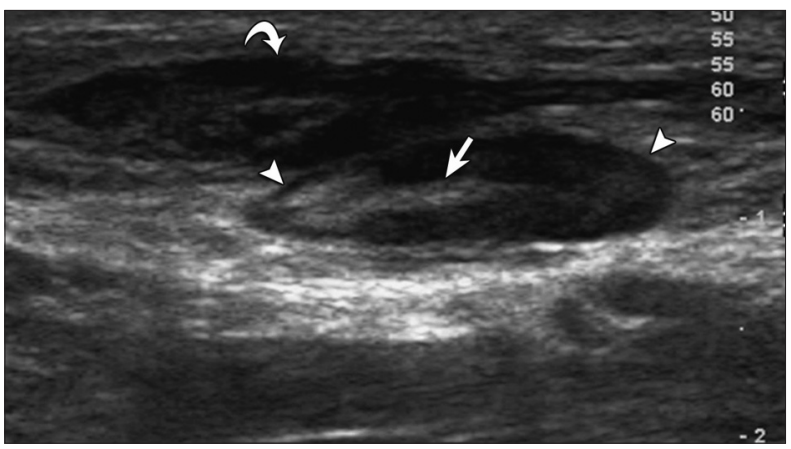

C

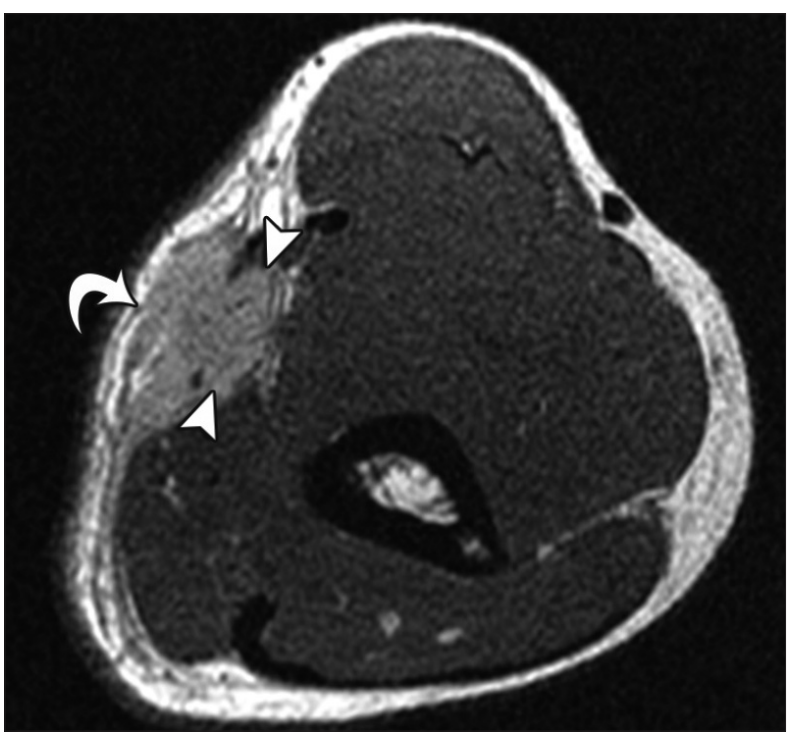

teristic of cat scratch disease, unlike other causes of lymph node enlargement.

Cat scratch disease usually presents with regional lymphadenopathy, painful swelling, and nonspecific clinical signs and symptoms as result of $B$ henselae infection. A definitive diagnosis is based on polymerase chain reaction for DNA analysis or serologic testing, as isolating Bartonella species in culture is difficult and usually unsuccessful; histologic examination reveals typical but nonspecific features, including granulomas with central necrosis at later stages. ${ }^{14,15}$

B

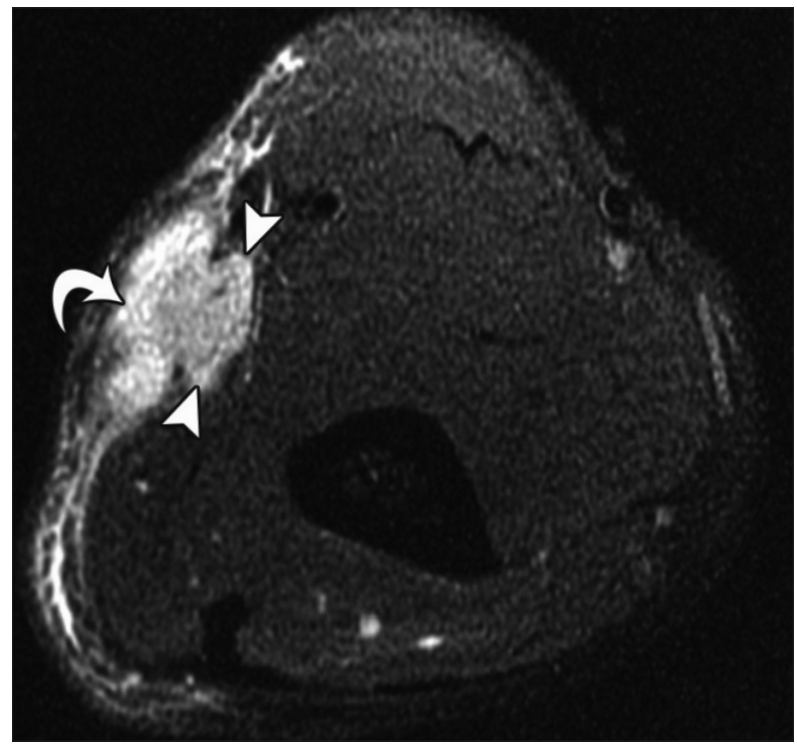

D

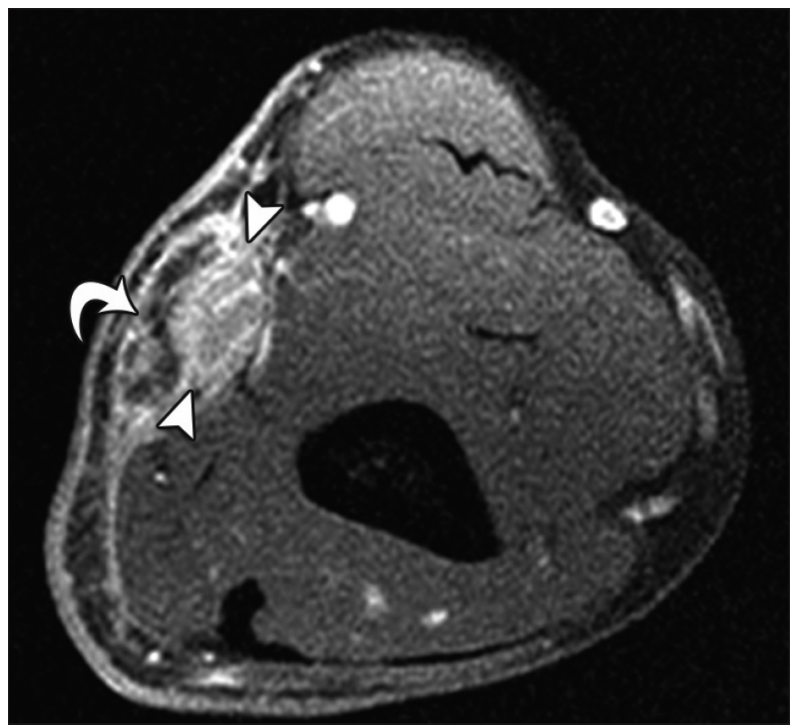


The presumptive diagnosis of cat scratch disease is frequently made on the basis of suggestive clinical features, including lymph node enlargement, cat exposure, and an upper extremity inoculation site. ${ }^{1}$ Nearly $75 \%$ of involved lymph nodes are located in the neck or upper extremity, most commonly at the medial epitrochlear region. 2,16

The peak age range for cat scratch disease is reported as between 2 and 14 years, and $40 \%$ of our study group ( 2 of 5) belonged to this age group., ${ }^{2,17}$ Between $95 \%$ and $99 \%$ of cat scratch disease cases follow contact with a cat, and approximately $75 \%$ report a cat scratch. ${ }^{5}$ In our study group, $80 \%$ (4 of 5) reported cat contact, with $40 \%$ ( 2 of 5 ) reporting a scratch. Our study group suggests that patients outside the peak age range and without a recalled history of a cat scratch may be more likely to undergo imaging. Atypical presentations may occur in $5 \%$ to $11 \%$ of patients, due to systemic dissemination, hematogenous osteomyelitis, or

Figure 4. Images from a 34-year-old man with a biopsy-proven hyperplastic lymph node. Grayscale long-axis sonogram (A) shows a hypoechoic mass (arrowheads) with a mixed pattern of hyperemia (not shown). T1-weighted (B), T2-weighted (C), and T1-weighted gadoliniumenhanced, fat-saturated (D) MR images show a corresponding soft tissue mass (arrows).

\section{A}

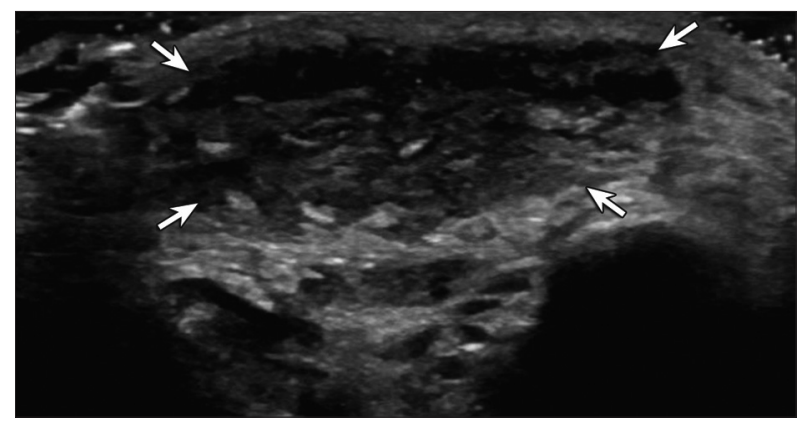

C

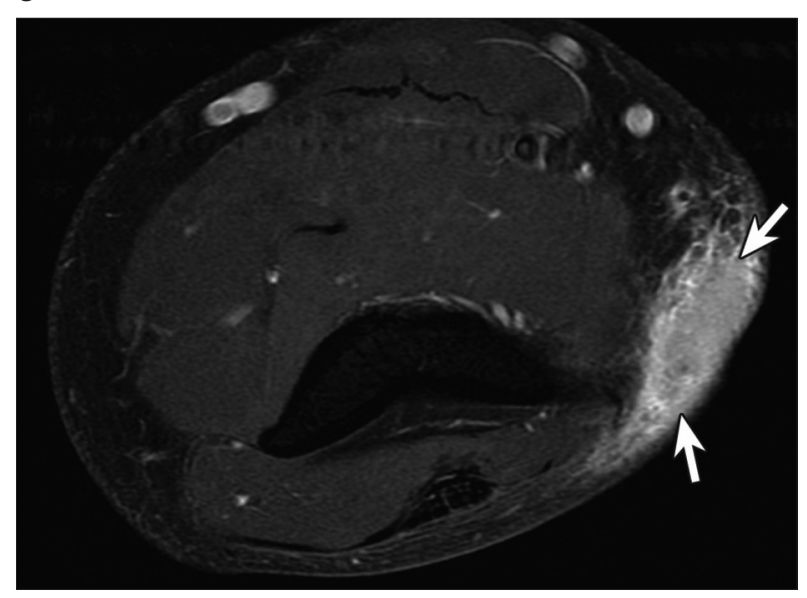

hepatosplenic involvement, and may occur more commonly in immune-compromised patients; however, none of our patients had clinical features to suggest this complication. ${ }^{2,3}$ Hepatosplenic involvement may be identified on sonography as multiple small hypoechoic nodules, which may eventually calcify. ${ }^{18,19}$ The presence of these lesions may further support the diagnosis of cat scratch disease, particularly when clinical suspicion for malignancy remains low. Most cases of cat scratch disease confined to the epitrochlear or axillary lymph nodes resolve within 3 months.

Epitrochlear nodes, which are also known as the cubital or supraepitrochlear lymph nodes, constitute part of the upper extremity superficial lymphatic system and are located in the subcutaneous tissue on the medial aspect of the elbow approximately 4 to $5 \mathrm{~cm}$ above the trochlea. ${ }^{20}$ Although the association of cat scratch disease with medial

B

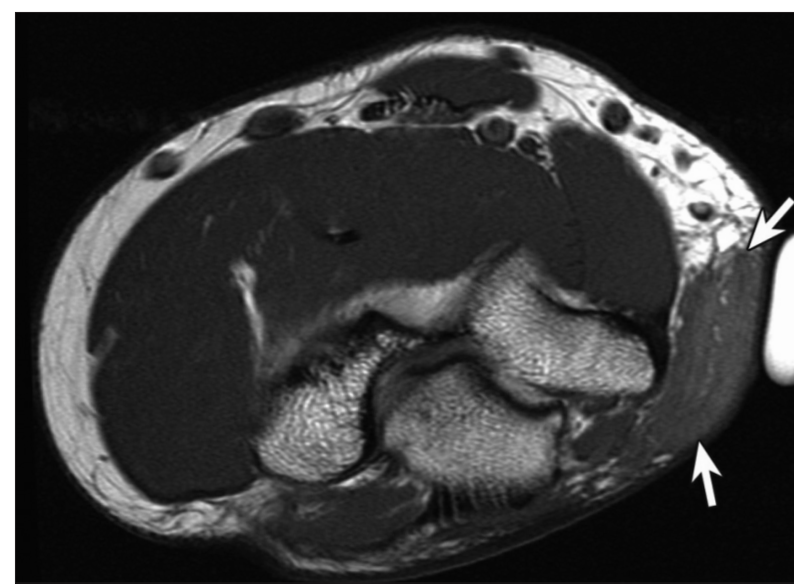

D

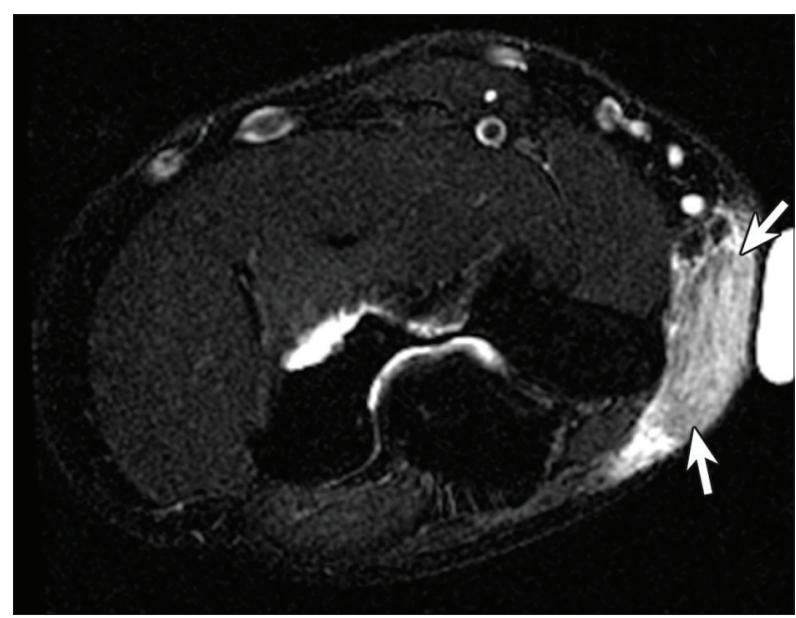


epitrochlear lymph node enlargement is established in the radiology and infectious disease literature, complex nodal masses with suppuration may mimic aggressive soft tissue masses, ${ }^{8,11}$ especially if specific lymph node features are not identified on MRI, which can occur when inflammation is extensive. Although the appearance of such lymph node lesions on MRI has been described, the sonographic features of cat scratch disease have not been fully evaluated. $^{10,12}$

Reported sonographic features of involved regional lymph nodes in cat scratch disease without suppuration include a hypoechoic mass with or without increased posterior through-transmission. ${ }^{12}$ Although the lymph nodes in our patients with cat scratch disease were also commonly hypoechoic ( $80 \%)$, those in patients with other causes of epitrochlear masses were also commonly hypoechoic (82\%). Similarly, the previously described increased posterior through-transmission was not seen in these cases as a specific feature, as this finding was present in $20 \%$ of masses from cat scratch disease and $25 \%$ from other causes.

Figure 5. Images from a 54-year-old man with a biopsy-proven epitrochlear mass from sarcoidosis. Grayscale (A) and color Doppler (B) long-axis sonograms show an oval symmetric hypoechoic mass (arrows) with a mixed pattern of hyperemia.

\section{A}

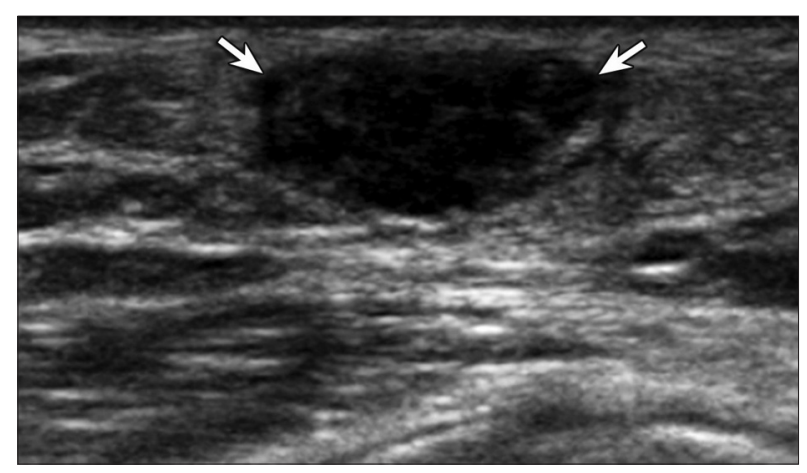

B

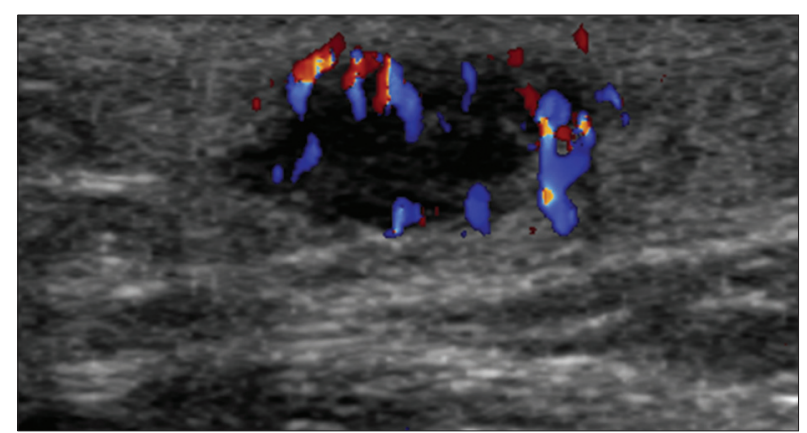

An additional finding, the presence of a markedly increased Doppler signal within involved nodes, has been previously identified as a suggestive feature of cat scratch disease, possibly related to neovascularization resulting from Bartonella infection, which may also produce vasoproliferative lesions involving the skin, brain, bone marrow, and mucosal surfaces. $^{21-23}$ The conspicuity of this feature on power Doppler imaging has been described as the "fire" pattern. ${ }^{12}$ In our study, central hyperemia was noted in $60 \%$ of cat scratch disease cases ( 3 of 5); however, hyperemia was present in $50 \%$ of non-cat scratch disease cases ( 8 of 16), although it was central in only $25 \%$.

Our retrospective review comparing the sonographic features of epitrochlear masses from cat scratch disease to other causes revealed 2 significant findings: asymmetry and the presence of an echogenic hilum. The cause of asymmetry is not clear but may explain the finding of an adjacent fluid collection, which was found to be substantial when comparing parameters as a function of a cat scratch disease versus non-cat scratch disease diagnosis and was present in $40 \%$ of cat scratch disease cases but only $6 \%$ of cases with other causes. The presence of an adjacent or intranodal fluid collection is believed to indicate nodal suppuration or necrosis, which is seen in up to $35 \%$ of cases. ${ }^{4,24}$ This appearance corresponds to the reported appearance of cat scratch disease on MRI, with mixed enhancing solid and fluid components (Figures 2 and 3 ). ${ }^{10}$ The other significant finding of a hyperchoic hilum seen in cat scratch disease was not unexpected; however, it was surprising that no other causes of an epitrochlear mass showed this finding, even the cases of lymph node hyperplasia.

This retrospective study had several limitations. Despite the frequency of cat scratch disease, there were only 5 patients with cat scratch disease undergoing sono-

Table 2. Analysis of Parameters as a Function of a Cat Scratch Disease Versus Non-Cat Scratch Disease Diagnosis

\begin{tabular}{ll}
\hline Parameter & Statistical Results \\
\hline Size & $t=0.217 ; P=.8301$ \\
Shape & $\chi^{2}=0.404 ; P=.8172$ \\
Symmetry & $\chi^{2}=8.750 ; P=.0031$ \\
Location & $\chi^{2}=0.5668 ; P=.5668$ \\
Multiplicity & $\chi^{2}=0.836 ; P=.3606$ \\
Echogenicity & $\chi^{2}=1.382 ; P=.5011$ \\
Hyperechoic hilum & $\chi^{2}=11.20 ; P=.0008$ \\
Peripheral anechoic/hypoechoic area & $\chi^{2}=3.544 ; P=.0500$ \\
Hyperemia & $\chi^{2}=0.153 ; P=.6959$ \\
Flow pattern & $\chi^{2}=5.565 ; P=.0619$ \\
Through-transmission & $\chi^{2}=0.053 ; P=.8188$ \\
\hline
\end{tabular}


graphic evaluations over a 13-year period, which reflects the fact that most of these cases are diagnosed on the basis of clinical and laboratory findings and are often not imaged. Due to the self-limiting nature of this condition, no cases of cat scratch disease underwent surgical excision, limiting pathologic correlation; however, positive diagnoses were obtained by serologic tests, clinical histories, and patient follow-up. In addition, as sonographic examinations were performed as part of routine patient care, there is intrinsic variability in the technique between sonographers; however, there is less operator dependence when imaging a soft tissue mass compared to imaging complex anatomic structures such as the rotator cuff. Last, interobserver and intraobserver variability of sonographic findings were not assessed.

In summary, the results of our study show that the sonographic finding of epitrochlear lymph node enlargement due to cat scratch disease most commonly is that of a hypoechoic lobular or oval mass with central hyperemia and a possible adjacent fluid collection; however, the findings of an asymmetric shape and a hyperechoic hilum differentiate cat scratch disease from other etiologies. By recognizing the common sonographic appearance of cat scratch disease, an additional clinical history for cat exposure and serologic testing for $B$ henselae may be considered to avoid unnecessary biopsy or resection.

\section{References}

1. Florin TA, Zaoutis TE, Zaoutis LB. Beyond cat scratch disease: widening spectrum of Bartonella henselae infection. Pediatrics 2008; 121 :e1413e1425.

2. Carithers HA. Cat-scratch disease: an overview based on a study of 1200 patients. Am J Dis Child 1985; 139:1124-1133.

3. Rohr A, Saettele MR, Patel SA, Lawrence CA, Lowe LH. Spectrum of radiological manifestations of paediatric cat-scratch disease. Pediatr Radiol 2012; 42:1380-1384

4. Wang CW, Chang WC, Chao TK, Liu CC, Huang GS. Computed tomography and magnetic resonance imaging of cat-scratch disease: a report of two cases. Clin Imaging 2009; 33:318-321.

5. Margileth AM. Cat scratch disease. Adv Pediatr Infect Dis 1993; 8:1-21.

6. Luddy RE, Sutherland JC, Levy BE, Schwartz AD. Cat-scratch disease simulating malignant lymphoma. Cancer 1982; 50:584-586.

7. Nimityongskul P, Anderson LD, Sri P. Cat-scratch disease: orthopaedic presentation. Orthop Rev 1992; 21:55-59.

8. Fox BC, Gurtler RA. Cat-scratch disease mimicking rhabdomyosarcoma. Orthop Rev 1993; 22:1148-1149.

9. Lefkowitz M, Wear DJ. Cat-scratch disease masquerading as a solitary tumor of the breast. Arch Pathol Lab Med 1989; 113:473-475.
10. Gielen J, Wang XL, Vanhoenacker F, et al. Lymphadenopathy at the medial epitrochlear region in cat-scratch disease. Eur Radiol 2003; 13:1363-1369.

11. Dong PR, Seeger LL, Yao L, Panosian CB, Johnson BL Jr, Eckardt JJ. Uncomplicated cat-scratch disease: findings at CT, MR imaging, and radiography. Radiology 1995; 195:837-839.

12. Garcia CJ, Varela C, Abarca K, Ferres M, Prado P, Vial PA. Regional lymphadenopathy in cat-scratch disease: ultrasonographic findings. Pediatr Radiol 2000; 30:640-643.

13. Barr LL, Kirks DR. Ultrasonography of acute epitrochlear lymphadenitis. Pediatr Radiol 1993; 23:72-73.

14. Regnery RL, Olson JG, Perkins BA, Bibb W. Serological response to "Rochalimaea henselae" antigen in suspected cat-scratch disease. Lancet 1992; 339:1443-1445.

15. Bass JW, Vincent JM, Person DA. The expanding spectrum of Bartonella infections, II: cat-scratch disease. Pediatr Infect Dis J 1997; 16:163-179.

16. Margileth AM. Dermatologic manifestations and update of cat scratch disease. Pediatr Dermatol 1988; 5:1-9.

17. Zangwill KM, Hamilton DH, Perkins BA, et al. Cat scratch disease in Connecticut: epidemiology, risk factors, and evaluation of a new diagnostic test. NEngl J Med 1993; 329:8-13.

18. Talenti E, Cesaro S, Scapinello A, Perale R, Zanesco L. Disseminated hepatic and splenic calcifications following cat-scratch disease. Pediatr Radiol 1994; 24:342-343.

19. Larsen CE, Patrick LE. Abdominal (liver, spleen) and bone manifestations of cat scratch disease. Pediatr Radiol 1992; 22:353-355.

20. Catalano O, Nunziata A, Saturnino PP, Siani A. Epitrochlear lymph nodes: anatomy, clinical aspects, and sonography features. Pictorial essay. JUltrasound 2010; 13:168-174.

21. Spinella A, Lumetti F, Sandri G, Cestelli V, Mascia MT. Beyond cat scratch disease: a case report of Bartonella infection mimicking vasculitic disorder. Case Rep Infect Dis 2012; 2012:354625

22. Conley T, Slater L, Hamilton K. Rochalimaea species stimulate human endothelial cell proliferation and migration in vitro.J Lab Clin Med 1994; 124:521-528

23. Stockmeyer B, Schoerner C, Frangou P, Moriabadi T, Heuss D, Harrer T Chronic vasculitis and polyneuropathy due to infection with Bartonella henselae. Infection 2007; 35:107-109.

24. Margileth AM, Hayden GF. Cat scratch disease: from feline affection to human infection. NEnglJ Med 1993; 329:53-54. 